\title{
Morphometric and gross anatomy studies of gonads in day-old Arabic chick (Gallus turcicus) lead to gonadal asymmetry
}

\author{
Iswati Iswati ${ }^{1, *}$ and Gatot Ciptadi $^{2}$ \\ ${ }^{1}$ Politeknik Pembangunan Pertanian Malang, 65200, Indonesia \\ ${ }^{2}$ Faculty of Animal Science, Universitas Brawijaya, Malang, 65145, Indonesia
}

\begin{abstract}
The differentiation of avian gonads occurred since the embryonic stage, resulted in asymmetric morphology. This study aimed to analyse the morphometric and gross anatomy of gonads in Day old Arabic chick. This study utilized 116 Day old Arabic chick. After necropsy, they were divided into 61 male and 55 female. The variables studies were length, width, volume, location, colour, and shape of the gonads. Data analysis used descriptive analysis and independent $T$ test. The results showed a significant difference $(\mathrm{P}<0.01)$ between the right and left ovary lengths $1.67 \pm 0.08 \mathrm{~mm}$ and $3.69 \pm 0.05$, respectively. A significant difference $(\mathrm{P}<0.01)$ between the width ovaries $0.64 \pm 0.03 \mathrm{~mm}$ (right ovary) and $1.18 \pm 0.03 \mathrm{~mm}$ (left ovary), respectively. It was found a significant difference $(\mathrm{P}<0.05)$ between the length of right and left testis $2.47 \pm 0.08$ $\mathrm{mm}$ and $3.29 \pm 0.07 \mathrm{~mm}$, and between volume of testis $1.46 \pm 0.12 \mathrm{~mm}^{3}$ (right testis) and $2.03 \pm 0.13 \mathrm{~mm}^{3}$ (left testis). However, the right and left testes width was not significant $(\mathrm{P}>0.01)$. The testes colour was creamy white, sometimes pink, and grey to black, the colour of ovaries was creamy yellow and pink. Morphometric and gonadal morphology of Day old Arabic chick showed gonadal asymmetry, and the left side is larger than the right side.
\end{abstract}

\section{Introduction}

Arabic chicken is one of the native chickens, layer-type chicken with superior egg production [1]. Its production potential has attracted farmers, so it has grown widely in several areas in Indonesia. In addition to having high egg production, Arabic chickens can adapt to various environmental conditions. Arabic chicken also does not have the brooding trait to extend their egg-laying period $[2,3]$.

Freshly hatched Arabic chickens are generally monomorphic, difficult to determine between males and females because they have almost the same phenotypic characteristics. Recently, research has been developed about sexing Day-old-chick in Arabic chickens to distinguish sex quickly and accurately. Research on Day-old Arabic chick sexing requires

\footnotetext{
*Corresponding author: iswati@polbangtanmalang.ac.id
} 
proof of sex as soon as possible. One of the proofs is through gonad identification (postmortem) in day-old chicks because they are relatively more available than molecular sexing [4]. Gonads are reproductive organs, ovaries to produce ova in females, and testes to produce spermatozoa in males [5]. Because the size of the gonads of freshly hatched chickens is relatively small, it is necessary to observe their morphology to determine the testes or ovaries.

The avian gonads have location and morphology differences from mammals; birds' testes are in the abdominal cavity, normally, the right ovary regresses. The left ovaries have been identified as functional gonad, and asymmetric development of the right and left ovaries has been reported since the embryonic phase [6]. The male, both gonads develop into testes [7]. Testicular asymmetry is also common in poultry, especially in passerines, and the degree of asymmetry decreases with the increasing age of chickens. The right and left testes are functional, although the left is larger in most species [8]. The size of the testes is often associated with their reproductive ability [9]. Testicular morphometric studies have been carried out on adult Nigerian local chickens under physiological conditions to predict the reproductive ability of the testes [10]. Left-Right (L:R) asymmetry could be detected in both sexes in chickens [11].

Based on the description, it is necessary to reveal the shape and size of the ovary and testes of Day-old Arabic chick. This study aimed to analyse the morphometric and gross anatomy of the testes and ovaries of Day-old Arabic chick. Descriptions and information about their morphological characters are essential to confirm gonadal observations for purposes of proving to sex, to predict male quality through post-hatching development, and detect the gonadal abnormalities in Day-old chick (DOC).

\section{Materials and methods}

\subsection{Day-old-chick Arabic chicken}

This study used 116 Day-old Arabic chick, resulting in mating between hen and Arabic gold rooster in previous studies [4]. Day-old chick obtained from the chicken breeding unit in Politeknik Pembangunan Pertanian Malang. The research was conducted at the Poultry Unit and the Reproduction Laboratory of the Politeknik Pembangunan Pertanian Malang.

The chicks have been estimated for their sex by feather sexing, then were euthanized by cervical dislocation. The necropsy procedures incise the ventral abdomen until the peritoneal cavity opens and removes the viscera organs, so the gonads are visible. The testes and ovaries were not removed from the abdominal cavity and were not weighed because relatively very small. The chicken was fixed in a dorsal lying position with the gonads still attached to the abdominal cavity, observed with direct eyes and then clarified under a trinocular stereo microscope connected to a computer to identify the gonads is easier.

\subsection{Research variable}

The gonads were examined for colour, shape, and location. Ovarian morphometrics includes the length and width of the right and left ovaries, while the testes include length, width, and volume. Data collection on the shape, colour, and location of the gonads was carried out under a stereo microscope with 10x magnification. The stereo microscope used is the Euromax SB 1903-P series produced by Holland with a DC 5000P camera. Measurement of the length and width of the gonads using the micrometre software from the 
camera. Quantitative data on testicular length and width were tabulated to calculate testicular volume.

Length of testes and ovaries was measured from the anterior end to the posterior end of the gonad, and gonad width was measured at the broadest part of the gonad. Testis's shape is ellipsoid [9], so the testicular volume was calculated using formula $=(4 / 3) \times \pi \times(a)^{2}(b)$. Where (a) is: half of the width and (b) is half of the length [12] or with formula (simplified) namely testicular volume $=0.523 \times(\text { Width })^{2}$ (Length) $[8]$.

\subsection{Statistical analyses}

The results of the gross anatomy identification of testes and ovaries were shown with explanation descriptions. The morphometric data were analysed using descriptive analysis. Differences in size between the right and left gonads were analysed by independent T-test at significance $\mathrm{P}<0.01$, using SPSS version 16. Data was presented in mean \pm SEM.

\section{Results and discussion}

\subsection{Gonads overview of day-old Arabic chick}

Freshly hatched chicks were identified to be 55 females and 61 males after necropsy. The testes and ovaries were located in the abdominal cavity and could be seen when the viscera organs were removed. Results of observations to the ovaries and testes morphology are shown in Figure 1. Postmortem examination of chick testes and ovaries showed that the gonads consist of two right and left at above the surface of the kidney. The gonads were connected to the backbone on the surface of the two kidneys.
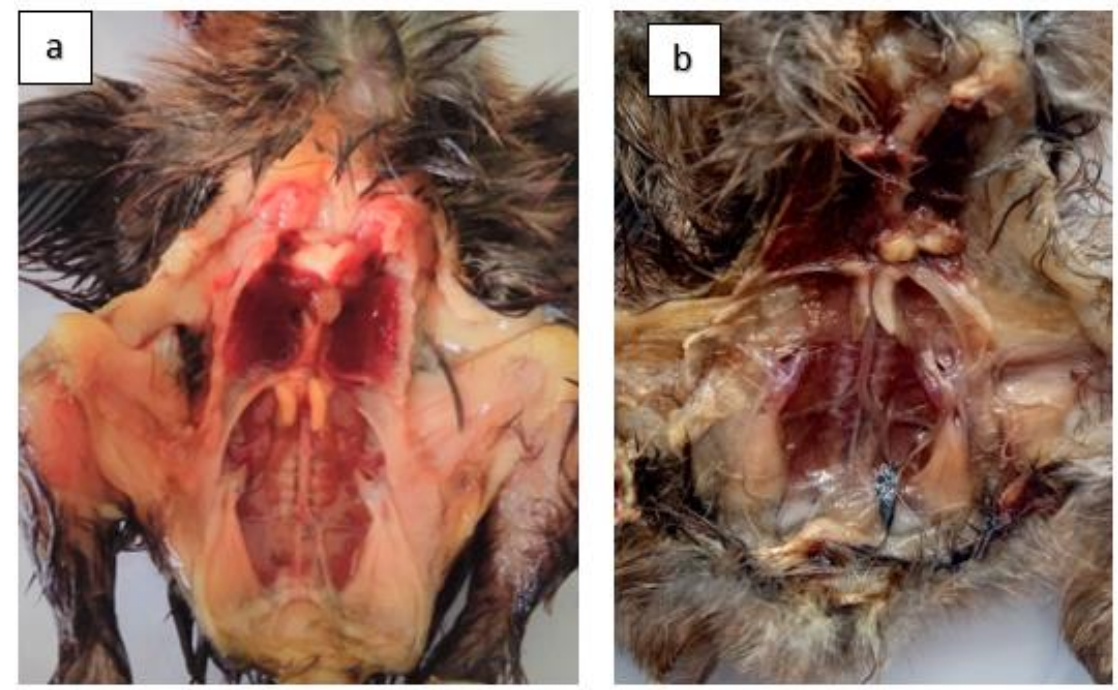

Fig. 1. Showing Gonads of Day-old Arabic chick a). Male chick ; b) Female chick. 


\subsection{Morphometric and Gross Anatomy of Day-old Arabic Chick Ovaries}

Gross anatomy observation of the gonads showed that the shape of the ovary is irregular, and the left ovary has a more defined shape than the right ovary. The ovaries are close to the adrenal glands, where the right side is caudolateral of the adrenal gland, while the left side covers the ventral adrenal gland. The ovaries are located in the ventromedial mesonephros although the mesonephros is sometimes not visible due to regression.

In general, the ovaries of Day-old Arabic chick have an elongated shape with slightly curved laterally or towards the kidneys. The left ovary shows a curvature towards the right kidney with various shapes and uneven edges. The right ovary is smaller with an elongated and irregular shape. Almost all of the left adrenal glands are covered by the ovaries, and the right adrenal gland is more visible. The colour of the ovaries is creamy yellow, transparent pink, and sometimes grey. The surface of the left ovary appears smoother, slightly compact. This ovarian character is still similar to the morphology of the ovaries of 1-week old chickens, as described by [6]. The gross anatomy of the ovary of Day-old Arabic chick was shown in Figure 2.
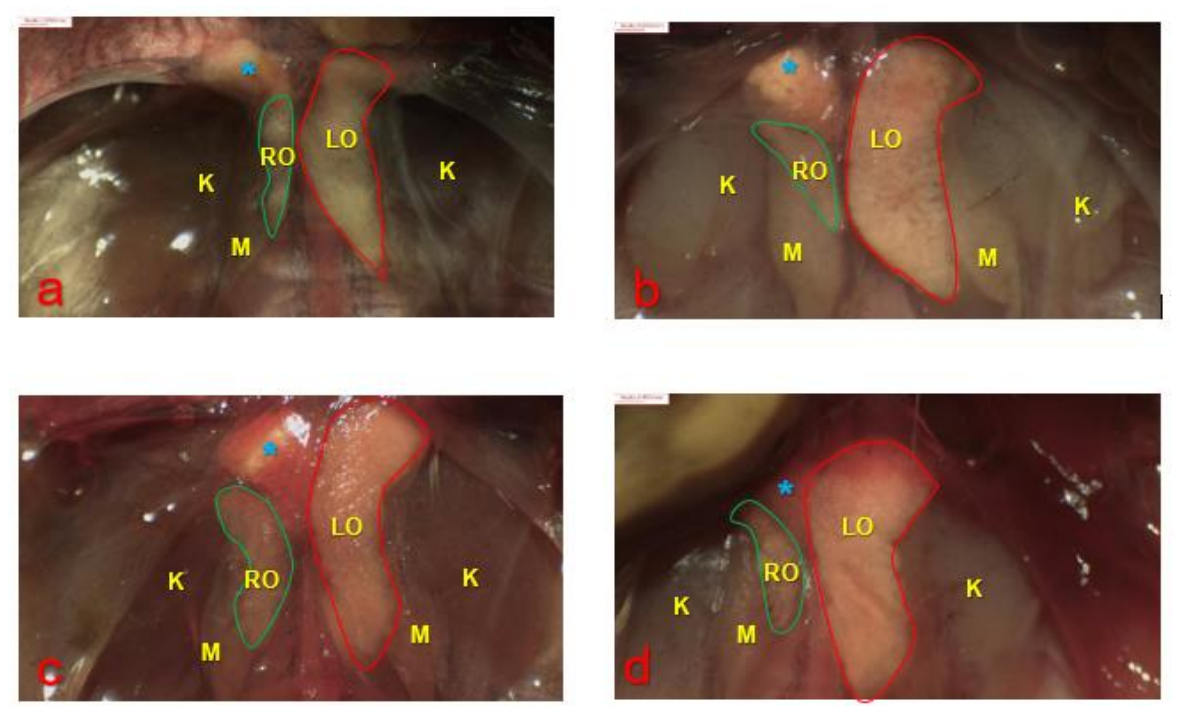

Fig. 2. Gross anatomy of ovaries Day-old Arabic chick. RO=Right ovary; LO=Left ovary; $\mathrm{K}=$ kidney; $\mathrm{M}=$ mesonephros; Blue $(*)=$ Adrenal Gland. The images were taken using a Euromax SB 1903-P stereo microscope and a DC 5000P camera, magnification: 10x, scale: $0.655 \mathrm{~mm}$.

Figure 2, part (a) the shape of the right ovary: small elongated, the right mesonephros appears small, and the left mesonephros is not visible; (b). the left ovary is much larger, the right and left mesonephros are still visible clearly; (c) the right ovary is like a bean arch, the right, and left mesonephros are still visual even though they are smaller; (d). The right ovary is small, like a bean arch and the adrenal gland and the right mesonephros are not visible. The difference between the right and left ovaries: the left ovary is more elongated than the right ovary, anterior end of the left ovary is wider than the posterior end. The left ovary appears thinner than the right ovary. 
Table 1. Ovaries Morphometric of the Day-old Arabic chick (Average \pm SEM) and T-test result.

\begin{tabular}{|l|c|c|}
\hline Characteristics & Right ovary & Left ovary \\
\hline Length of ovary (mm) & $1.67 \pm 0.08$ & $3.69 \pm 0.05$ \\
\hline Width of ovary (mm) & $0.64 \pm 0.03$ & $1.18 \pm 0.03$ \\
\hline Longest ovary size (mm) & 3.34 & 4.55 \\
\hline Shortest ovary size (mm) & 0.66 & 2.23 \\
\hline Difference in length (left-right) of the ovary (mm) & \multicolumn{2}{|c|}{$0.97 \pm 0.09$} \\
\hline Difference in width (left-right) of the ovary (mm) & \multicolumn{2}{|c|}{$0.58 \pm 0.06$} \\
\hline The T-test between right and left ovary length & \multicolumn{2}{|c|}{$P$-value $<0.0001^{* *}$} \\
\hline The T-test between the width of the right and left ovaries & \multicolumn{2}{|c|}{$P$-value $<0.0001^{* *}$} \\
\hline
\end{tabular}

Note: $* *$ : indicates a significant difference $(\mathrm{P}<0.01)$

Table 1 shows that the length and width of the ovaries vary widely. T-test analysis showed that the size difference between the right and left ovaries was very significant $(\mathrm{P}<0.01)$. The size difference of the chick ovary results from the asymmetry process that has occurred since the embryonic phase. In domestic birds, the left and right ovaries begin to grow around 72 hours of initial incubation [13] and morphological differentiation of gonads occurred on day 6.5 of incubation [5]. In the early stages before sexual differentiation, the ovaries of chicken embryos develop symmetrically without showing significant left-right asymmetry. As the embryo develops, the right ovary in the medulla develops without a cortex. This cortical development is considered essential for developing the left ovary [13]. There is a size difference between the right and left ovaries, resulting in ovarian asymmetry on the 20th day of incubation [6] [14]. From this study, ovary asymmetry in Arabic chicken was identified after hatching. The hen has only one ovary and oviduct on the left side [5].

\subsection{Morphometric and Gross Anatomy of Day-old Arabic chick testes}

Gross anatomy observations showed the testes are located in the ventromedial mesonephros. The mesonephros has regressed from the embryonic phase at hatching. It appears as a small part at the bottom of the gonads. The invasion of primordial germ cells forms embryonic gonads into the coelomic epithelium that partially covers the mesonephros [15]. The cranial portion of the testes is located close to the ventral border of the lungs. In contrast, the caudal part is located in the cranial-ventral kidney.

The colour of the testes in Arabic chicken was creamy white, slightly pink, grey, and some were black in the left or right testicles, or even black in both right and left testes. The testicles of certain breeds of fowl may be mixed with black colour and white patches or maybe entirely greyish black due to the presence of the pigment melanin in the melanoblasts, in the connective tissue of the testes [14]. The gonads of sexually immature or resting birds (in seasonal birds) have yellowish-white to black or grey colouration, becoming moderately black in regressing testes and containing melanoblasts [14]. Testicular colour variability can be caused by sexual activity in testes and chicken's age. Bright yellow in juveniles and grey-white in adults of the quails and ostrich. The bright yellow colour in juveniles is due to the accumulation of lipids in the interstitial cells and the grey-white colour in adults due to the spread of interstitial cells by enlarging seminiferous tubules after sexual maturity [20]. The results of this study, most of the testicle's colour in DOC Arab chickens is yellow-white (creamy), the findings of the other colours besides 
bright yellow, still have to be confirmed histologically to support the determination of its quality.

The description of the location, shape, and colour of the Day-old Arabic chick testes is presented in Figure 3.
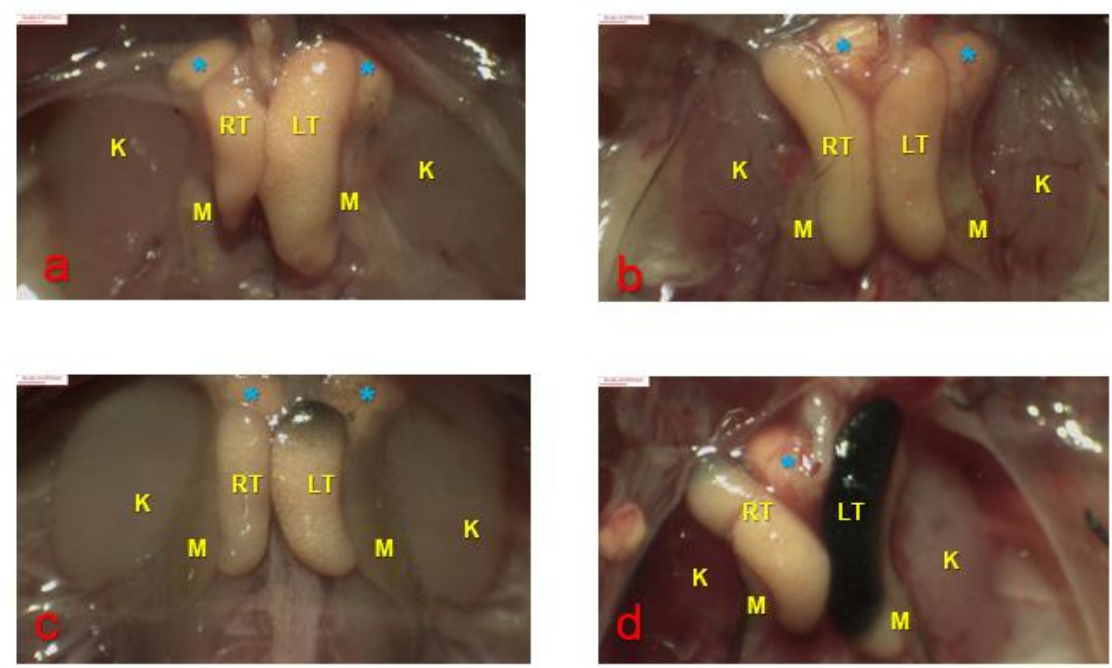

Fig. 3. Gross anatomy of Day-old Arabic chick testes. R= Right testes; LT= Left testes; $\mathrm{K}=$ kidney; $\mathrm{M}=$ mesonephros; Blue (*): Adrenal Gland. The images were taken using a Euromax SB 1903-P stereo microscope and a DC 5000P camera, magnification: 10x, scale: $0.655 \mathrm{~mm}$.

The testes of birds in this study are bean-shaped and elongated ovals, generally belonging to the category of an ellipsoid or prolate spheroid shape [8]. The form of the testes tends to be straight, lengthwise cranial and caudal, or slightly curved towards the kidney. Figure 3 shows that (a) the left testis is larger than the right testis, the right and left sides show clear mesonephros; (b) the size of the right testis is larger than the left testis; (c). The length of the right testis is almost the same as that of the left. However, the left testis is bigger and grey with a wider anterior end and black colour (d). the left testis is longer than the right testis, most of the left testis is darker in colour, and the anterior end of the right testis is grey. The results on Day-old Arabic chick, the number of detected black colour on the testes was $22.95 \%$. Melanoblasts cause dark colour in the interstitium, so the testes are melanistic. Bicoloured testes are not considered gonads abnormal. The testes with bicolour do not show any histologic abnormalities in African flamingos [16]. In this study, the position of the right and left testes tends to be symmetrical, but there are differences in size. The morphometrics of Day-old Arabic chick is presented in Table 2.

Table 2 showed the size (length, width and volume) of left testis is larger than right testis. The results of the independent T-test analysis showed that the length and volume of the testes were significantly different between the right and left testes $(\mathrm{P}<0.01)$. In contrast, the testes' width was not significantly different between the right and left $(\mathrm{P}>0.01)$. This study showed that the number of Day-old Arabic chick with the left testis was larger than the right, namely $72.13 \%$, and the number of Day-old Arabic chick with the right testis was larger than the left testis only $27.86 \%$. It has been described in previous study that the left testis larger than the right in birds commonly, but there are exceptions in some species where there is no difference in size, and the right testicle is larger than the left testis [14]. The left testicle is larger than the right testis in Gallus domesticus is $63 \%$ in birds and $57 \%$. 
The right testis is larger than the left testis, only $26.5 \%$, while both testes are the same in $8.2 \%$ of birds [8].

Table 2. Testes Morphometric of Day-old Arabic chick (Average \pm SEM)

\begin{tabular}{|l|c|c|}
\hline Characteristics & Right testis & Left testis \\
\hline Length of the testis (mm) & $2.47 \pm 0.08$ & $3.29 \pm 0.07$ \\
\hline Width of testis (mm) & $0.98 \pm 0.03$ & $1.04 \pm 0.02$ \\
\hline The volume of the testis $\left(\mathrm{mm}^{3}\right)$ & $1.46 \pm 0.12$ & $2.03 \pm 0.13$ \\
\hline Difference in length (left-right) of the ovar (mm) & \multicolumn{2}{|c|}{$0.82 \pm 0.08$} \\
\hline Difference in width (left-right) of the ovar (mm) & \multicolumn{2}{|c|}{$0.05 \pm 0.03$} \\
\hline Volume total of testis ( $\left.\mathrm{mm}^{3}\right)$ & \multicolumn{2}{|c|}{$3.49 \pm 0.22$} \\
\hline T-test between right and left testis length & P-value $0.001 * *$ \\
\hline T-test between right and left testis width & \multicolumn{2}{|c|}{ P-value $0.194 *$} \\
\hline T -test between the volume of right and left testis & \multicolumn{2}{|c|}{ P-value $0.002 * *$} \\
\hline
\end{tabular}

Note: $*=$ not significant difference; $* *=$ a significant difference $(\mathrm{P}<0.05)$.

Testicular asymmetry may be caused by irregular migration of the Primordial Germ Cells (PGC) to the left and right sides during embryonic development. This testicular asymmetry has been reported that the right gonadal cortex loses its chemotactic attraction to PGCs. Thus, it leads to the left gonadal cortex on day 3th of embryogenesis [14]. Similar results were reported by [11] and [18] that the number of PGCs in the left testis is more than in the right testis, and in the chicken embryos on the 6th day of incubation, the size of the left testis relatively larger. Despite the lack of a relationship between male quality and the degree of testicular asymmetry, but several studies have found that testicular asymmetry increases with age [19]. The results of this study can be used as a basis for Day-old chick testicular morphometrics to be compared with the age afterward in the same type of chicken.

\section{Conclusion}

Studies of Gonad morphometrics in Day-old Arabic chick have shown gonadal asymmetry, left ovary are larger than the right ovary. The size and volume of the left testis are larger than the right testis. Gross anatomy and evidence of gonadal asymmetric in Arabic chicken can be used as reference when observing the gonads and as information on the gonad's development at hatching. Further research is needed to reveal the histological and molecular asymmetry, and the observation of gonadal size at a later age, which can be associated with the quality of the chicken.

Thank you to the Head of the Agricultural Human Resources Extension and Development Agency, Ministry of Agriculture as the scholarship provider. The Director of Politeknik Pembangunan Pertanian Malang has been permitted to use research facilities, especially the Poultry Livestock Installation and Reproduction Laboratory.

\section{References}

1. R. Hartawan, N.L.P.I. Dharmayanti, HAYATI J. Biosci. 23, 160-167 (2016).

2. T. Sartika, K. Diwyanto, S. Iskandar, Mengenal plasma nutfah ayam Indonesia dan 
pemanfaatannya, (Balai Peneliti Ternak, Sukabumi, Indonesia, 2007).

3. G. Indra, Achmanu;, A. Nurgiartiningsih, Junal Ternak Tropika. 14, 8-14 (2013).

4. Iswati, M.H. Natsir, G. Ciptadi, T. Susilawati, Journal of Animal \& Plant Sciences, 44, 7708-7716 (2020).

5. S. Guioli, S. Nandi, D. Zhao, J. Burgess-Shannon, R. Lovell-Badge, M. Clinton, Sex. Dev. 8, 227-242 (2014).

6. J.D.L. Mfoundou, Y.J. Guo, M.M. Liu, X.R. Ran, D.H. Fu, Z.Q. Yan, M.N. Li, X.R. Wang, Poult. Sci. 100, 101191 (2021).

7. R. Tóth, B. Lázár, Á. Südy, A. Nagy, A. Kidane, M. Anand, E. Gócza, N. Biotechnol. 33, S212 (2016).

8. G.R. Graves, The Auk. 121, 473 (2004).

9. N.A. Bachmid, F.Y. Purba, A.M.S. Apada, D.K. Sari, IOP Conf. Ser. Earth Environ. Sci. 343 (2019).

10. A.K. Okpe Godwin Chidozie, Nwatu Ugochukwu, Anim. Res. Int. 7, 1163-1168 (2010)

11. S. Intarapat, O. Satayalai, Anat. Res. Int. 2014, 1-9 (2014).

12. A. Dawson, J. Avian Biol. 34, 19-123 (2003).

13. Y. Ishimaru, T. Komatsu, M. Kasahara, Y. Katoh-Fukui, H. Ogawa, Y. Toyama, M. Maekawa, K. Toshimori, R.A.S. Chandraratna, K.I. Morohashi, H. Yoshioka, Development. 135 (2008) 677-685.

14. B.G.M. Jamieson, Reproductive Biology and Phylogeny of Birds (CRC Press, Boca Raton, Florida, United States, 2011).

15. C.G. Scanes, Sturkie's Avian Physiology (Academic Press, San Diego, United States, 2015).

16. L. Crosta, H. Gerlach, M. Bürkle, L. Timossi, Exot. Anim. Pract. 6, 57-83 (2003).

17. S. Intarapat, C.D. Stern, J. Poult. Sci. 51, 352-358 (2014).

18. T.K. TriBudi, T. Kostaman, Majalah Ilmiah Peternakan, 23, 107-112 (2020).

19. T.R. Birkhead, K.L. Buchanan, T.J. Devoogd, E.J. Pellatt, T. Székely, C.K. Catchpole, Song, Anim. Behav. 53 965-971 (1997).

20. P. Dharani, S. Ushakumary, V. Sundaram, C. Joseph, G. Ramesh. Int. J. Morphol. 36, 909-914 (2018). 